INITIAL TIME DEPENDENCE OF ABUNDANCES IN SOLAR ENERGETIC PARTICLE EVENTS

D. V. Reames, C. K. Vg

Code 661, Laboratory for High Energy Astrophysics

NASA Goddard Space Flight Center

Greenbelt, MD 20771

and

A. J. Tylka

Code 7654, E. O. Hulburt Center for Space Research Naval Research Laboratory, Washington, DC 20375

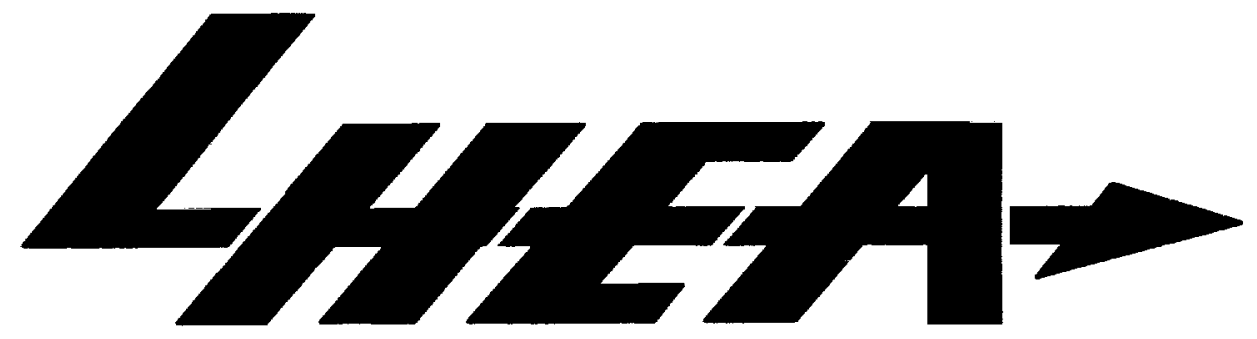

National Aeronautics and Space Administration Goddard Space Flight Center Greenbelt, Maryland 20771 


\title{
INITIAL TIME DEPENDENCE OF ABUNDANCES IN SOLAR ENERGETIC PARTICLE EVENTS
}

\author{
D. V. Reames, C. K. $\mathrm{Ng}^{1}$ \\ Code 661, NASA/Goddard Space Flight Center, Greenbelt, MD 20771 \\ reames@lheavx.gsfc.nasa.gov, cheeng@lheavx.gsfc.nasa.gov
}

and

A. J. Tylka

Code 7654, E. O. Hulburt Center for Space Research, Naval Research Laboratory, Washington, DC 20375

tylka@gamma.nrl.navy.mil

Accepted for publication in Astrophysical Journal (Letters)

${ }^{1}$ also Dept. of Astronomy, University of Maryland, College Park, MD 20742 



\begin{abstract}
We compare the initial behavior of $\mathrm{Fe} / \mathrm{O}$ and $\mathrm{He} / \mathrm{H}$ abundance ratios and their relationship to the evolution of the proton energy spectra in "small" and "large" gradual solar energetic particle (SEP) events. The results are qualitatively consistent with the behavior predicted by the theory of $\mathrm{Ng}$ et al. $(1999 \mathrm{a}, \mathrm{b})$. He/H ratios that initially rise with time are a signature of scattering by non-Kolmogorov Alfvén wave spectra generated by intense beams of shock-accelerated protons streaming outward in large gradual SEP events.
\end{abstract}

Subject headings: acceleration of particles - shock waves - Sun: particle emission Sun: corona 


\section{INTRODUCTION}

In recent years it has become clear that the particles in the "gradual" solar energeticparticle (SEP) events are accelerated at shock waves driven out from the Sun by fast coronal mass ejections (CMEs), and not in solar flares (Kahler et al. 1984; Kahler 1992, 1994; Gosling 1993; Reames 1995, 1997, 1999; Reames et al. 1996). Measurements of ionization states of Fe suggest that ambient (unheated) coronal material provides the seed population, even for particles that are shock-accelerated to energies up to $600 \mathrm{MeV} / \mathrm{amu}$ (Tylka et al. 1995).

In large SEP events, the theory of self-generated waves has been employed to understand both the acceleration of the particles at interplanetary shock waves in time equilibrium (Lee 1983) and the transport of particles outward from a source near the Sun (Ng \& Reames 1994; Reames \& Ng 1998). The scattering of particles by hydromagnetic waves is a time-dependent non-linear process in which particles can amplify or damp waves in such a way that the particle transport is drastically modified.

Recent papers by $\mathrm{Ng}$, Reames, \& Tylka (1999a, b) report on a dynamic simulation of the evolution in time and space of the particles and waves associated with an outbound shock. This model assumes that particles are injected with power-law spectra and coronal element abundances at the position of the shock, but then follows the subsequent evolution of the distribution functions of particles and waves in detail. As the particles stream out from the shock, different species at the same velocity are scattered differently depending upon their charge-to-mass ratios, $Q / A$. This model explained the observed (Tylka, Reames, \& Ng 1999a, b) systematic variations in element abundance ratios like $\mathrm{Fe} / \mathrm{O}$, for example, in terms of differential scattering of $\mathrm{Fe}$ and $\mathrm{O}$ in the proton-generated Alfvén waves. However, a truly surprising feature of the model of $\mathrm{Ng}$ et al. $(1999 \mathrm{a}, \mathrm{b})$ was its prediction of the initial behavior of $\mathrm{He} / \mathrm{H}$.

Ions resonate with waves of wave number $k_{r e s}=B / \mu P$, where $\mathrm{B}$ is the magnetic field strength, $\mu$ is the ion's pitch angle, $P=p c / Q e$ is its magnetic rigidity, and $p$ its momentum. When ions of given velocity propagate through a background Kolmogorov wave spectrum with a $k^{-5 / 3}$ dependence, those with higher $Q / A$ are scattered more. Hence, the earliest arrivals, with $\mu \sim 1$, will be the species with highest rigidity (lowest $Q / A$ ) and a ratio such as $\mathrm{Fe} / \mathrm{O}$ will begin at high values and then decline with time. Any abundance ratio of highrigidity/low-rigidity ions at the same velocity, including $\mathrm{He} / \mathrm{H}$, should behave in the same way, beginning at high values and declining with time. However, early observers (e.g. Witte et al. 1979; Mason et al 1983) were surprised to find that ratios of the fitted time profiles of $\mathrm{He}$ and $\mathrm{H}$ occasionally rose initially as a function of time. These observers admitted that they did not understand the behavior. However, they did not compare the spectra of events with rising and falling $\mathrm{He} / \mathrm{H}$ and the phenomenon was not reexamined when more-sensitive instruments became available.

According to $\mathrm{Ng}$ et al. (1999a, b) the early rise can occur because the earliest protons, of $2 \mathrm{MeV}$, for example, have yet to generate waves, but $2 \mathrm{MeV} / \mathrm{amu} \mathrm{He}$ resonates with waves created by $\sim 8 \mathrm{MeV}$ protons that arrived much earlier. Obviously, this effect will occur only if there are "enough" $\sim 8 \mathrm{MeV}$ protons to matter, i.e. in "large" events. 
Furthermore, the effect will be most pronounced for magnetically well-connected events because their rapid onsets magnify differences in transport.

We note that the addition of flare-accelerated particles, with abundances such as enhanced $\mathrm{Fe} / \mathrm{O}$, can also affect the initial ratios (Reames 1990). However, with the new calculations of $\mathrm{Ng}$ et al. (1999a, b) it now seems more likely that most of the early peaks in $\mathrm{Fe} / \mathrm{O}$ result from transport rather than from the presence of an additional flare source. In any case, flares are extremely unlikely to contribute material with suppressed $\mathrm{He} / \mathrm{H}$.

In this paper, we compare some observational examples of the initial behavior of abundance ratios such as $\mathrm{He} / \mathrm{H}$ and $\mathrm{Fe} / \mathrm{O}$ in "large" and "small" events and we examine the related proton spectra that define the meaning of event size. In the comparison, we use observations from the Wind, ISEE 3, and IMP 8 spacecraft.

\section{INITIAL ABUNDANCE BEHAVIOR AND PROTON SPECTRA}

Figure 1 compares the time dependence of hourly-averaged intensities and abundances of several ion species in the gradual SEP events of 1998 May 6 and 1998 September 30. Peak proton intensities are more than an order of magnitude larger, depending on energy, in the second event than in the first. Both events show a decline in $\mathrm{Fe} / \mathrm{O}$ as a function of time. However, while the May 6 event shows a rapid early decline in $\mathrm{He} / \mathrm{H}$, especially at low energies, the event of September 30 shows a dramatic early rise in $\mathrm{He} / \mathrm{H}$ lasting about $8 \mathrm{hrs}$ at the lowest energies.

We examine the evolution of the proton energy spectra in the two events in Figure 2. Times of the 2-hr averaged spectra labeled A-D in Figure 2 are spaced at 4-hr intervals and are noted along the time axis of Figure 1. In contrast to the steep spectra in the May 6 event, the spectra in the large September 30 event appear to be affected by the streaming limit (Ng \& Reames, 1994; Reames \& Ng 1998) out to at least $10 \mathrm{MeV}$ for $\sim 12$ hours. During this period there are as many protons at $\sim 8 \mathrm{MeV}$ as at $2 \mathrm{MeV}$ at $1 \mathrm{AU}$ in this large event, so it is not surprising to see appreciable scattering and suppression of low-energy He that persists for several hours. Of course, it is the cumulative effect of the protongenerated waves from the source to the observer that increases the scattering of He and reduces $\mathrm{He} / \mathrm{H}$. The shape of the local proton spectrum merely exposes this same complex underlying behavior. According to the theory ( $\mathrm{Ng}$ et al. 1999a, b), the actual amplitude and duration of the rise phase in $\mathrm{He} / \mathrm{H}$, as for other ratios such as $\mathrm{Fe} / \mathrm{O}$, depends upon several parameters, including the injected intensity at the shock and the shock compression ratio and their radial dependences.

To find additional events with clear unobstructed onsets, we have returned to the historic database. In Figure 3 we compare two such events, both from W50. While $\mathrm{Fe} / \mathrm{O}$ ratios are more poorly determined by ISEE 3 than by Wind, the events in Figure 3 show the same qualitative behavior as those in Figure 1. In fact, the rather complex behavior of $\mathrm{Fe} / \mathrm{O}$ in the 1978 September 23 event is very similar to that previously reported for the 1998 April 20 event by Tylka et al. (1999a). The latter event also has rising $\mathrm{He} / \mathrm{H}$.

Proton energy spectra for the 1978 September 23 event in Figure 4 again show the pronounced flattening out to $10-20 \mathrm{MeV}$ that are characteristic of streaming-limited spec- 
tra. Spectra for the 1981 April 24 event are beginning to show limiting only at the lowest energies.

\section{CONCLUDING REMARKS}

The initial rise in $\mathrm{He} / \mathrm{H}$ is a sign of non-Kolmogorov Alfven-wave spectra that are evolving with time. Proton spectra that are initially flattened at low energies and enhanced at high energies are consistent with the generation of initially steepened wave spectra that preferentially scatter $\mathrm{He}$ relative to $\mathrm{H}$ in the onsets of large SEP events. The flattened spectra are themselves the product of streaming-limited transport (Lee, 1983; $\mathrm{Ng} \mathrm{\&}$ Reames 1994; Reames \& Ng 1998; Reames 1999). The rising $\mathrm{He} / \mathrm{H}$ ratios probe the cumulative effect of these steepened wave spectra from the shock out to $1 \mathrm{AU}$; they add another unique feature to the growing list of observational consequences of particlegenerated waves. In an era of measurement of rare elements and isotopes, it is ironic that proton observations can still provide fundamental new understanding.

AJT was supported by NASA DPR-S92662F during the course of this work. 


\section{REFERENCES}

Gosling, J. T. 1993, J. Geophys. Res., 98, 18949.

Kahler, S. W. 1992, ARA\&A, 30, 113.

Kahler, S. W. 1994, ApJ, 428, 837.

Kahler, S. W., Sheeley, N. R., Jr., Howard, R. A., Koomen, M. J., Michels, D. J., McGuire R. E., von Rosenvinge, T. T., \& Reames, D. V. 1984, J. Geophys. Res. 89, 9683.

Lee, M. A. 1983, J. Geophys. Res., 88, 6109.

Mason, G. M., Gloeckler, G., and Hovestadt, D. 1983, ApJ 267, 844.

Ng, C. K., \& Reames D. V. 1994, ApJ 424, 1032.

Ng, C. K., Reames, D. V., \& Tylka, A. J. 1999a, Geophys. Res. Lett. 26, 2145.

Ng, C. K., Reames, D. V., \& Tylka, A. J. 1999b, Proc. $26^{\text {th }}$ Internat. Cosmic Ray Conf. (Salt Lake City) 6, 151.

Reames, D. V. 1990, ApJ (Letters) 358, L63.

Reames, D. V. 1995, Revs. Geophys. (Suppl.) 33, 585.

Reames, D. V. 1997, in: Coronal Mass Ejections, edited by N. Crooker, J. A. Jocelyn, J. Feynman, Geophys. Monograph 99, (AGU press) 217.

Reames, D. V. 1999, Space Sci. Revs., 90, 413.

Reames, D. V., Barbier, L. M., \& Ng, C. K. 1996, ApJ, 466, 473.

Reames, D. V., \& Ng, C. K. 1998, ApJ, 504, 1002.

Tylka, A. J., Boberg, P. R., Adams, J. H., Jr., Beahm, L. P., Dietrich, W. F., \& Kleis, T. 1995, ApJ (Letters), 444, L109.

Tylka, A. J., Reames, D. V., \& Ng, C. K. 1999a, Geophys. Res. Lett. 26, 2141.

Tylka, A. J., Reames, D. V., \& Ng, C. K. 1999b, Proc. $26^{\text {th }}$ Internat. Cosmic Ray Conf. (Salt Lake City) 6, 135.

Witte, M., Wibberenz, G., Kunow, H., \& Muller-Mellin, R. 1979, Proc. $16^{\text {th }}$ Internat. Cosmic Ray Conf., (Kyoto) 5, 79. 


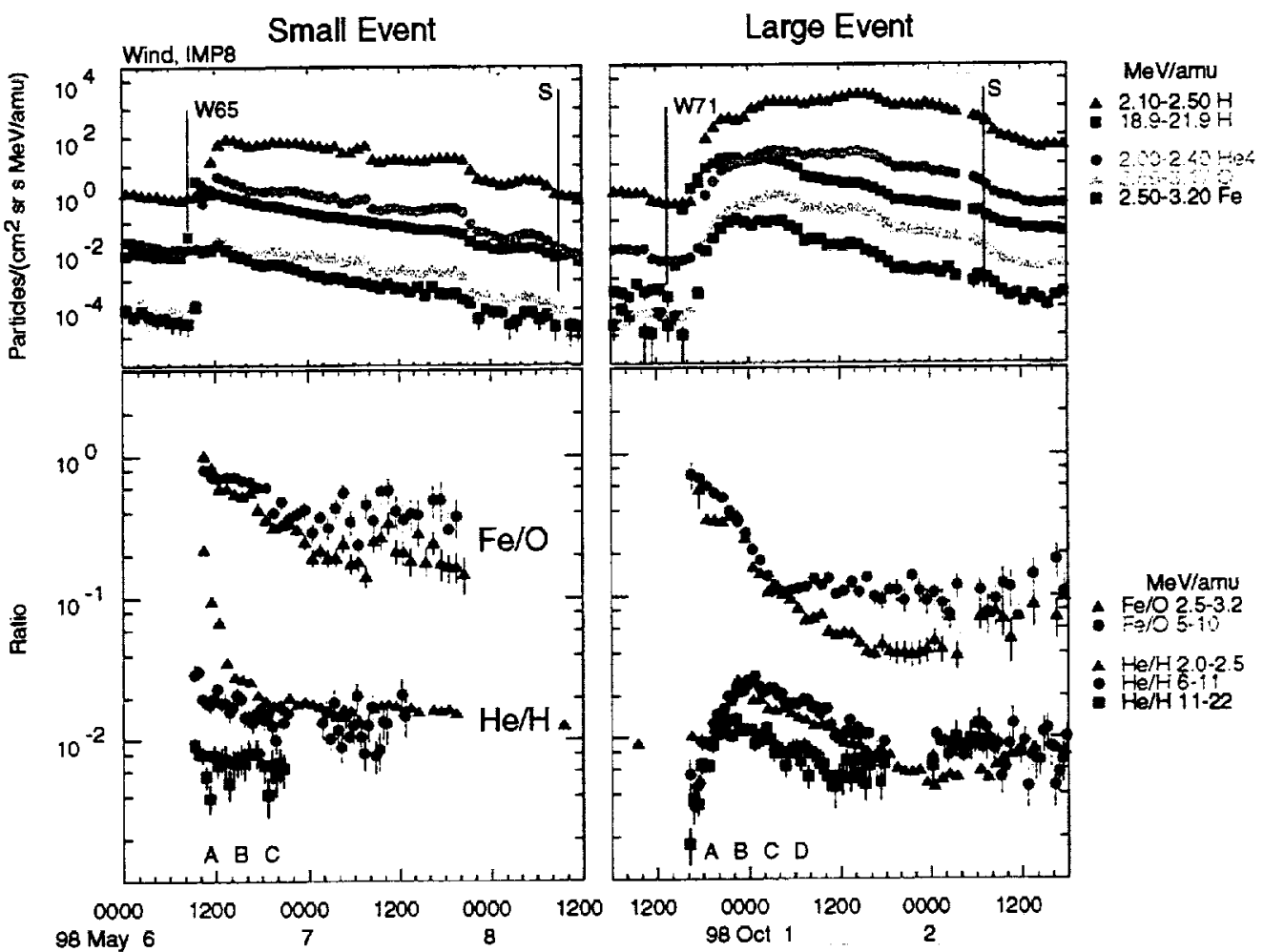

Fig. 1. Intensities and abundances of ion species vs. time are compared for the small 1998 May 6 SEP event and the large 1998 September 30 event. The initial rise in $\mathrm{He} / \mathrm{H}$ and corresponding fall in $\mathrm{Fe} / \mathrm{O}$ are clear in the September 30 event.

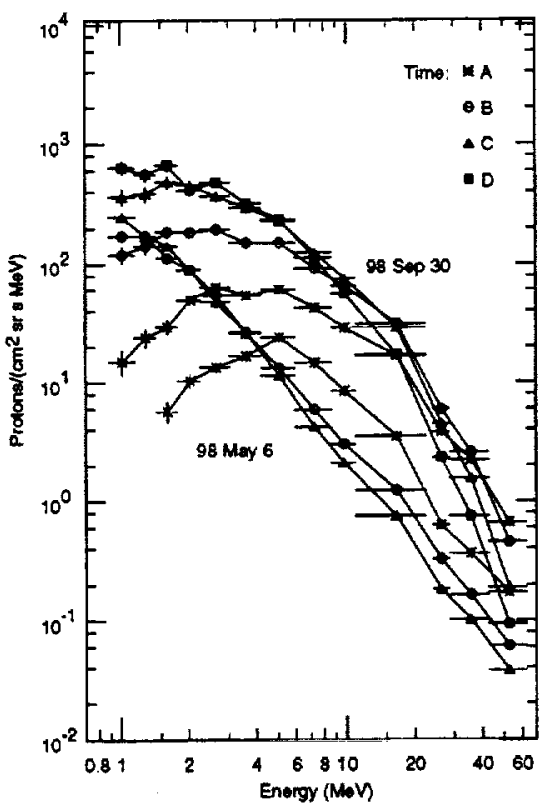

Fig. 2. Proton energy spectra are shown at several times, labeled A-D, corresponding to the times noted along the time axis in Figure 1. 


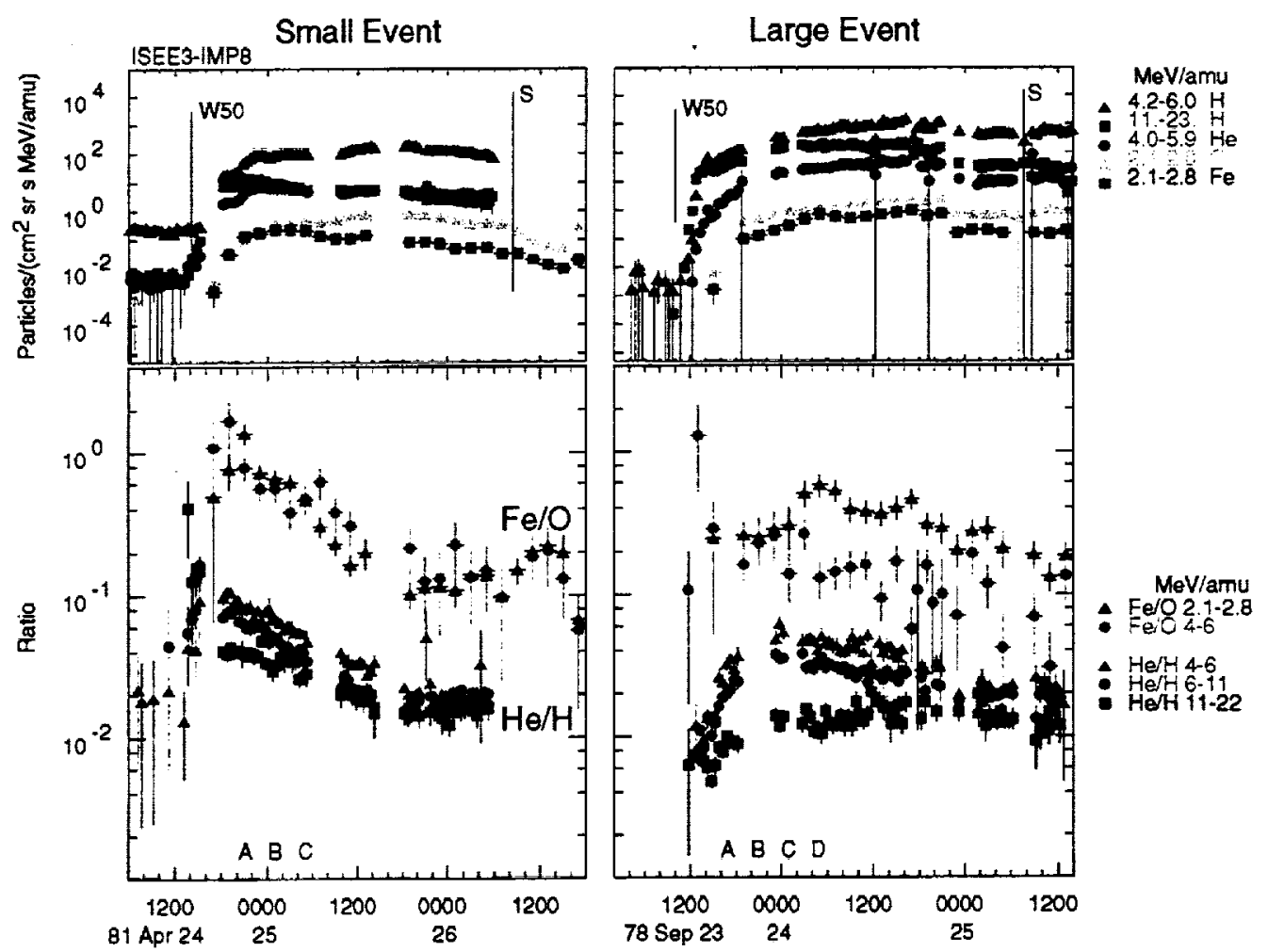

Fig. 3. Intensities and abundances of ion species $v s$. time are compared for the small 1981 April 24 SEP event and the large 1978 September 23 event.

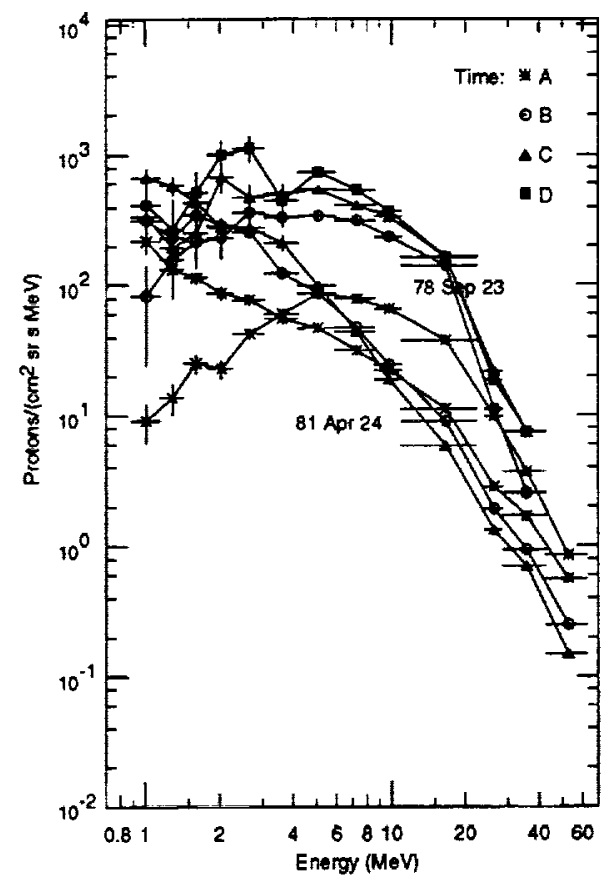

Fig. 4. Proton energy spectra are shown at several times, labeled A-D, corresponding to the times noted along the time axis in Figure 3. 